\title{
Thin films with high surface roughness: thickness and dielectric function analysis using spectroscopic ellipsometry
}

\author{
Daniel Lehmann ${ }^{*}$, Falko Seidel and Dietrich RT Zahn
}

\begin{abstract}
An optical surface roughness model is presented, which allows a reliable determination of the dielectric function of thin films with high surface roughnesses of more than $10 \mathrm{~nm}$ peak to valley distance by means of spectroscopic ellipsometry. Starting from histogram evaluation of atomic force microscopy (AFM) topography measurements a specific roughness layer (RL) model was developed for an organic thin film grown in vacuum which is well suited as an example. Theoretical description based on counting statistics allows generalizing the RL model developed to be used for all non-conducting materials. Finally, a direct input of root mean square (RMS) values found by AFM measurements into the proposed model is presented, which is important for complex ellipsometric evaluation models where a reduction of the amount of unknown parameters can be crucial. Exemplarily, the evaluation of a N,N'-dimethoxyethyl-3,4,9,10-perylene-tetracarboxylic-diimide (DiMethoxyethyl-PTCDI) film is presented, which exhibits a very high surface roughness, i.e. showing no homogeneous film at all.
\end{abstract}

Keywords: Ellipsometry; Roughness; Thickness; AFM; RMS; Dielectric function; PTCDI

\section{Background}

Spectroscopic ellipsometry (SE) is a commonly used tool to determine the thickness of thin films and investigate their dielectric function in a non-destructive way. During SE measurements the reflection properties of the whole sample are recorded. A subsequent evaluation by modeling the measured data is necessary to extract properties of individual layers. This procedure requires some knowledge of the general sample structure: the dielectric function of the substrate should be known with high precision and also the order of layers (e.g. substrate/oxide/film 1/film 2/ roughness) is a necessary input parameter. The more parameter values are unknown in the beginning of the evaluation, the more complex is the evaluation and eventually no solution might be found if some unknown parameters are highly correlated. A possible solution is a step by step analysis of the sample during the preparation, i.e. by recording SE spectra after growth of each individual layer. As roughness is a side effect of layer growth itself, this procedure cannot be used for the determination of

\footnotetext{
* Correspondence: daniel.lehmann@physik.tu-chemnitz.de Semiconductor Physics, Technische Universität Chemnitz, 09107 Chemnitz, Germany
}

surface roughness. Another way of reducing the number of unknown parameters is the inclusion of results from other techniques as, for example, UV-vis spectroscopy. Here, we report on the inclusion of AFM topography measurements to support the evaluation of SE spectra of thin film samples with high surface roughness. We then generalize the RL model to be useful for any evaluation of surface roughness of thin films grown on substrates. Finally, it is shown how the root mean square (RMS) determined by AFM measurements can directly be used as input parameter during SE evaluation.

\section{Methods}

The common way to handle a rough film in ellipsometric modeling is to divide it into two parts: one part containing the homogeneous film and the other part representing a non-homogeneous form referred to as "roughness layer" (Figure 1a).

The first part is simulated with the physically meaningful dielectric function belonging to the material. The determination of this dielectric function is usually the aim of the evaluation once the layer thicknesses have been determined. The second part is typically simulated using an

\section{Springer}




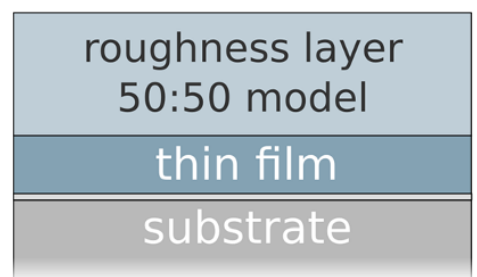

(a)

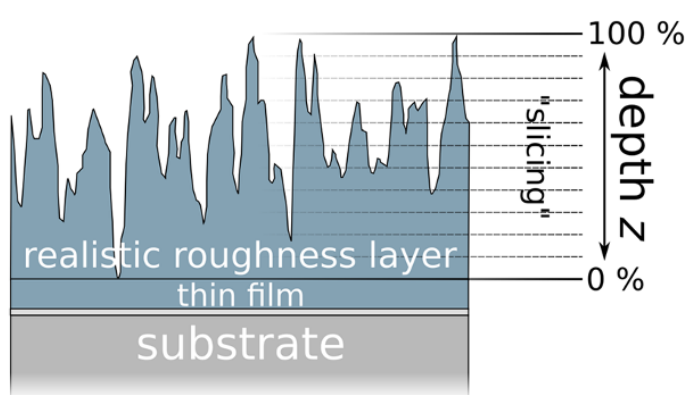

(b)

Figure 1 Roughness layer model comparison. (a) Common ellipsometry evaluation model: the RL is treated as an effective medium approximation (EMA) of 50\% material and 50\% void. (b) Realistic roughness layer derived from a cross section through an AFM picture of a DiMethoxyethyl-PTCDI film (Figure 2a). By z-slicing the RL into infinitesimal thin slices and treating each slice with an individual EMA material ratio, a more precise $\mathrm{RL}$ model can be derived.

effective medium approximation (EMA), a mixture of 50\% material - having the dielectric function of the first part of the simulated layer - and of $50 \%$ void space with the dielectric function of vacuum (further referred to as "50:50 model") (Gordan et al. 2006a, b; Louis et al. 2007; Volintiru et al. 2008). However, this estimation of the mixture ratio is not precise. The realistic roughness topography of grown layers has a profile comparable to mountains: if one virtually cuts a volume segment into slices of different height, at the height of the valleys the amount of material in each slice is high. At the height of the mountain tips, however, the amount of material is almost zero in each slice (Figure 1b). Hence, the material to void ratio is not constant throughout the roughness part of the layer, but changes as a function of distance to the virtual interface between the RL and the homogeneous part. A similar approach was already discussed by Aspnes et al. (1979), but with various geometrically constructed roughness profiles instead of AFM measured profiles.

The consequence of using a simple 50:50 model is an incorrect dielectric function of the roughness part and, hence, an incorrect dielectric function of the homogeneous part as both parts are correlated during the evaluation. These inaccuracies are small for transparent thick films with a small surface roughness. However, if the thickness of the RL has the same order of magnitude as the homogeneous film thickness, a reliable determination of the dielectric function is not possible.

AFM is a method to determine the topography of a sample surface. Commonly, an RMS value is given as value for the surface roughness. For determination details see Méndez-Vilas et al. (2002). However, much more information can be deduced for creating a sophisticated roughness model for SE. Exemplarily, an extreme case should be discussed: the dielectric function of an organic thin film of DiMethoxyethyl-PTCDI with a very high surface roughness is determined. A more detailed view on this semiconducting molecule itself and the implication of high surface roughness on electrical behavior when used in organic field-effect transistors can be found elsewhere (Lehmann and Zahn 2009). The thin films were prepared by organic molecular beam deposition under high vacuum conditions $\left(p_{\text {base }}<5 \times 10^{-7} \mathrm{mbar}\right.$ ) on Silicon $/ \mathrm{SiO}_{2}(1.4 \mathrm{~nm})$ substrates. The nominal thickness, respectively the deposited mass, during growth was monitored by a quartz micro balance (QMB). A nominal thickness of $30 \mathrm{~nm}$, based on known QMB ratios for other PTCDI derivatives forming smooth films, was the deposition target. The SE measurement was performed 47 days after the film deposition using a Woollam VASE Ellipsometer, measuring at three incidence angles $\left(65^{\circ}\right.$, $70^{\circ}, 75^{\circ}$ ) in a spectral range from $0.74 \mathrm{eV}$ to $4.50 \mathrm{eV}$. The depolarization was measured additionally using an M2000 T-Solar Ellipsometer from Woollam in the same spectral range. Figure 2a shows an AFM picture of DiMethoxyethyl-PTCDI. As comparison a second picture of another molecule, N,N'-diphenyl-3,4,9,10-perylenetetracarboxylic-diimide (DiPhenyl-PTCDI, nominally $30 \mathrm{~nm}$ thick), is also shown in Figure 2b. The comparison later demonstrates the error tolerance of the method with regard to a wrong input.

\section{Results and discussion}

For the method presented, the presumption has to be made that the Fresnel relations are valid. This is true for wavelengths much larger than the RL thickness and structure periodicity. Hence, the light scattering has to be weak and depolarization can be ignored. In the presented case the RL as well as the periodicity are in the range of $100 \mathrm{~nm}$. As the film and RL thicknesses are determined in the transparent region below $1.4 \mathrm{eV}$ $(\lambda \geq 886 \mathrm{~nm})$ we consider the Fresnel relations valid here. Figure 3 shows that the depolarization is low even for higher energies. 

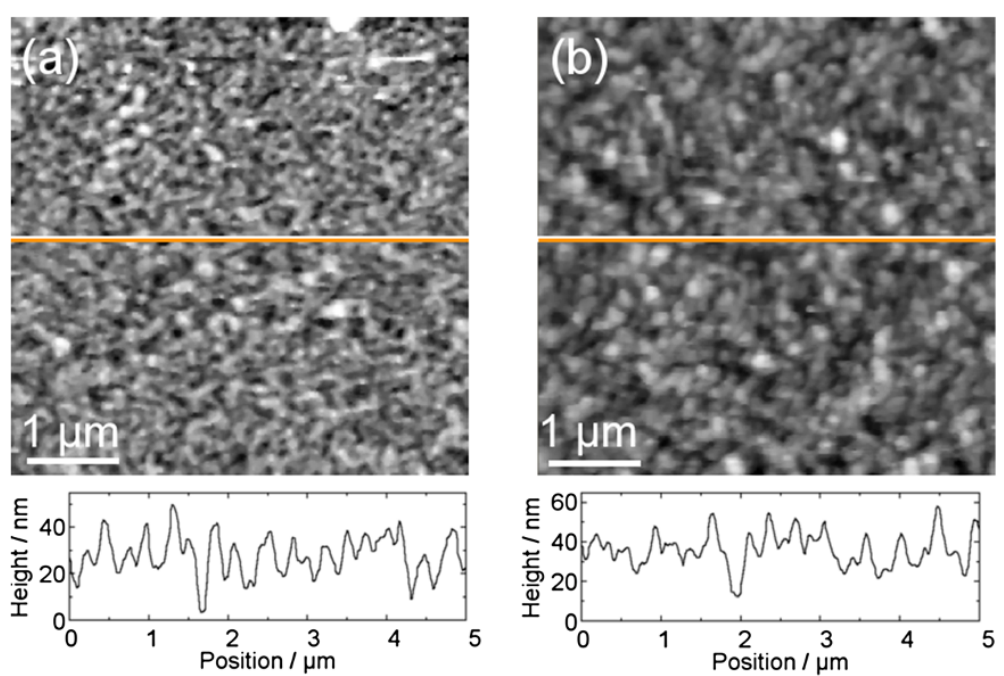

Figure 2 AFM measured topographies. (a) Height profile of DiMethoxyethyl-PTCDl: grayscale from $0 \mathrm{~nm}$ (black) to $58 \mathrm{~nm}$ (white), (b) height profile of DiPhenyl-PTCDI for comparison: grayscale from $0 \mathrm{~nm}$ (black) to $94 \mathrm{~nm}$ (white). The roughness parameter, i.e. the material/void function, is deduced by integrating over a grayscale histogram of each AFM picture.

Slicing the measured volume segment into equal height slices as described above can be performed by taking a histogram of the grayscale AFM pictures. Each grey level corresponds to a specific height slice. The amount of pixels with the same grey level determines the amount of material in the corresponding height slice. An integration over all grey levels leads to a material/ void ratio as a function of the height position within the RL (Figure 4).
The resulting material/void function can be fitted by the Boltzmann equation

$$
v(z)=\frac{A-B}{1+e^{(z-C) / D}}+B
$$

where $v(z)$ is the percentage of voids as a function of the dimensionless depth $z$, starting from the homogeneous film with $v(z=0 \%)=0 \%$. The depth is also given

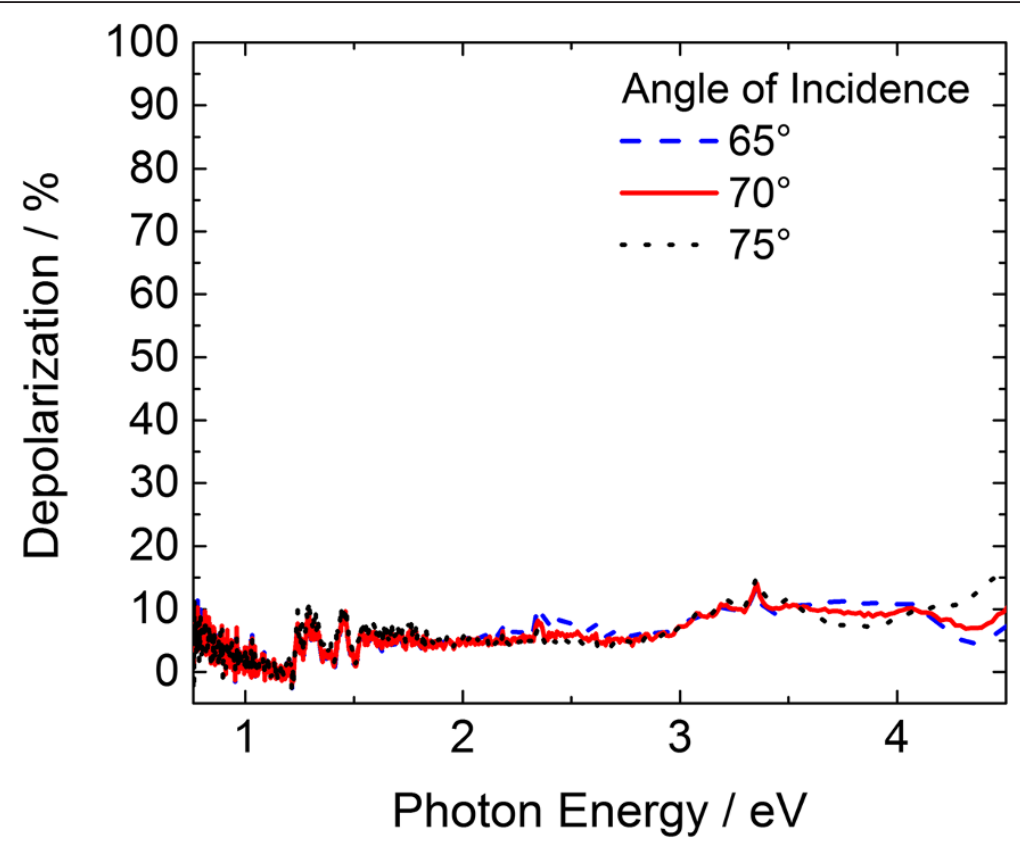

Figure 3 Depolarization of the DiMethoxyethyl-PTCDI sample during ellipsometry measurement. 


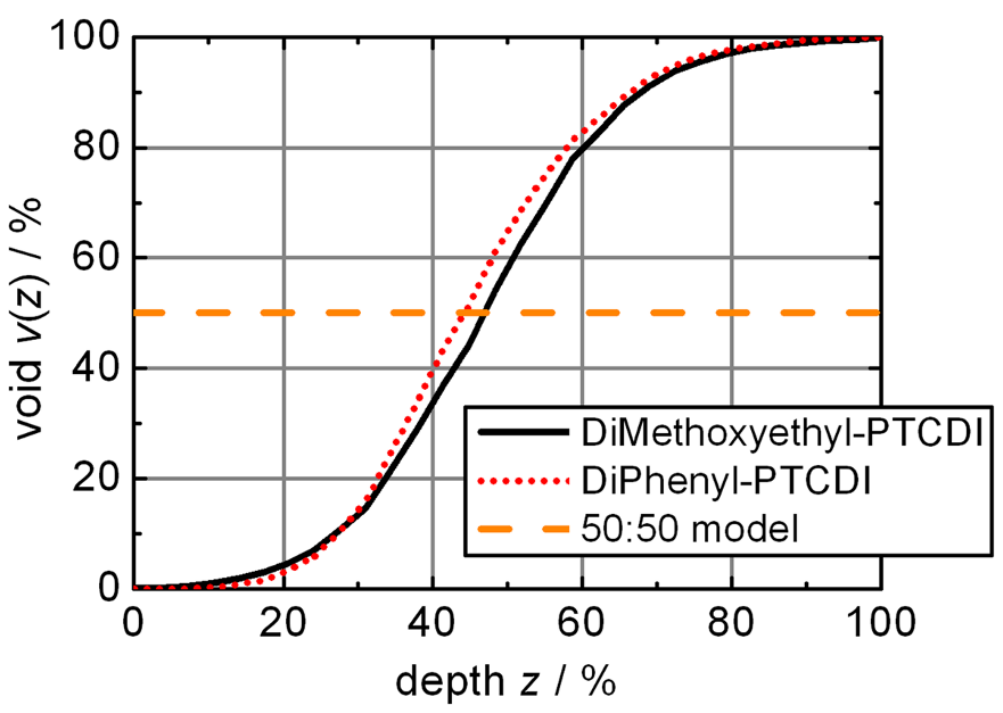

Figure 4 Material/void ratio comparison. DiMethoxyethyl-PTCDI and DiPhenyl-PTCDI ratios are deduced from the AFM topography measurements of Figure 1.

as percentage as the thickness of the film is variable during fitting while SE modeling is performed. The resulting parameters are compared in Table 1; they are similar for both films.

The Boltzmann equation with the determined parameters is transferred into an SE RL model of the layer utilizing a Bruggeman EMA (Bruggeman 1935) in the WVASE32 software (J.A. Woollam Co.). The homogeneous layer and RL thicknesses were determined using a Cauchy model in the range of $0.76 \mathrm{eV}$ to $1.40 \mathrm{eV}$. Table 2 compares the results of the deduced material/void functions (layer order: $\mathrm{Si} / \mathrm{SiO}_{2} /$ homogeneous film/AFM derived RL) to the results of the 50:50 model (layer order: $\mathrm{Si} / \mathrm{SiO}_{2} /$ homogeneous film/50:50 model RL).

The results from the material/void functions are very similar and predict both a Volmer-Weber type film growth with no homogeneous film below. The 50:50 model yields only uncertain values where a small change of starting parameters results in significantly different thicknesses. The peak valley distance - identical to the rough layer thickness in the material/void function model - is predicted to be larger than the AFM measured height profile. This can be explained with the limited resolution of the AFM tip: with a diameter of at least $10 \mathrm{~nm}$ only peaks and valleys of the same scale and above can be resolved.

Table 1 Boltzmann equation parameters determined by fitting the AFM derived material/void ratios

\begin{tabular}{lcccc}
\hline & A/ \% & B/ \% & C/ \% & D/ \% \\
\hline DiMethoxyethyl-PTCDI & -1.5 & 100.3 & 46.6 & 9.7 \\
DiPhenyl-PTCDI & -2.8 & 99.7 & 44.0 & 9.3 \\
\hline
\end{tabular}

Even though the model was derived from AFM pictures the depth $z$ was included as a percentage and not as a thickness value in order to be generally applicable. Therefore, the shape of the material/void function does not depend on the actual thickness. This is especially supported by the results of the DiPhenyl-PTCDI topography measurements: although the AFM height profile exhibits a peak-to-valley distance almost twice as large as for the DiMethoxyethyl-PTCDI, the derived material/ void function yields very comparable results during thickness determination in SE.

The determined thicknesses are used as input during the determination of the dielectric function. Therefore, a general oscillator model was utilized. Figure 5 shows the fits to $\psi$ and $\Delta$ for the 50:50 model and the presented AFM derived model. Figure 6 compares the results. Even though the quality of the fit does not differ significantly, the 50:50 model overestimates the dielectric function. Additionally, the error bar has to be considered to be as large as for the thickness determination: this result is not reliable. The material/void function model results

Table 2 Thicknesses $d$ of a homogeneous DiMethoxyethyl-PTCDI film below the surface roughness and the corresponding roughness layer heights $d_{r}$ determined for a film with the use of different roughness profiles

\begin{tabular}{lcc}
\hline Roughness profile of & $\boldsymbol{d} / \mathbf{n m}$ & $\boldsymbol{d}_{\mathbf{r}} / \mathbf{n m}$ \\
\hline DiMethoxyethyl-PTCDI (AFM) & 0 & $107.6 \pm 0.3$ \\
DiPhenyl-PTCDI (AFM) & 0 & $104.2 \pm 0.3$ \\
Proposed model with $\sigma=13.5 \%$ & 0 & $94.8 \pm 0.3$ \\
50:50 model & $27 \pm 76$ & $39 \pm 70$ \\
\hline
\end{tabular}



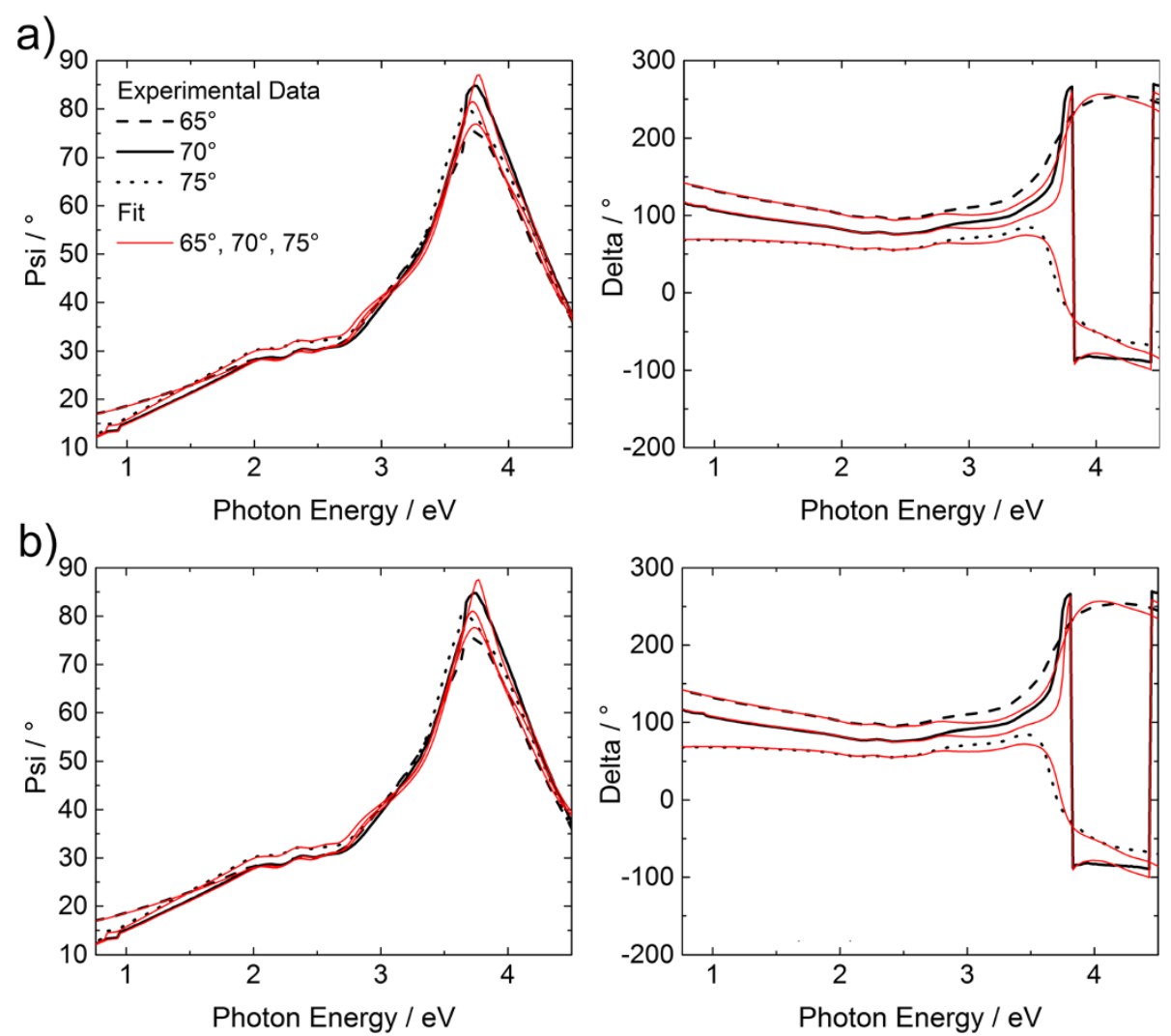

Figure 5 The ellipsometric angles $\psi$ and $\boldsymbol{\Delta}$ and the corresponding fits with general oscillator model. (a) Fit of the 50:50 model, (b) fit of the AFM derived roughness profile model.

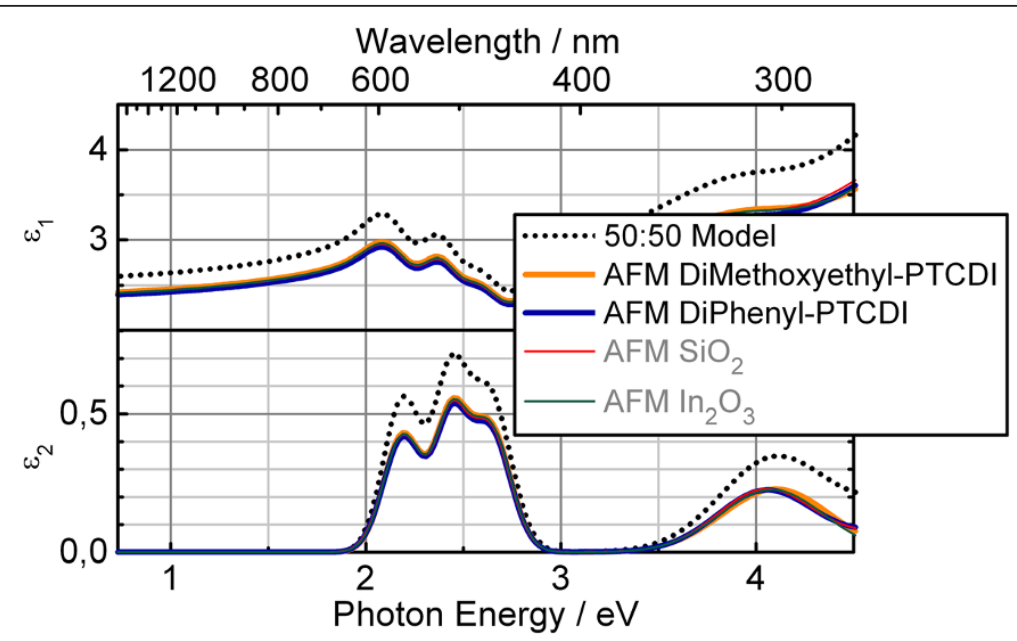

Figure 6 Dielectric functions determined for a DiMethoxyethyl-PTCDI rough thin film using different roughness profiles. Dotted curve: dielectric function determined using the conventional 50:50 EMA model. Orange line: dielectric function determined using the actual AFM profile of the film (parameters in Table 1). Other lines: dielectric functions for the same DiMethoxyethyl-PTCDI film, but using the AFM roughness profiles of completely different films. The AFM derived roughness profiles yield almost identical results independent of their manifold origin, whereas the common 50:50 model overestimates the dielectric function. 
for the dielectric functions deviate only slightly. The dielectric function of the AFM derived material/void function of DiMethoxyethyl-PTCDI itself is the most reliable one.

Not shown in detail are further stress tests of the material/void function model: AFM pictures of thin films with a large range of thicknesses and from various sources where converted to material/void functions as presented above. Exemplarily, results from $\mathrm{SiO}_{2}(1.23 \mathrm{~nm}$ peak to valley distance) $)^{a}$ and $\mathrm{In}_{2} \mathrm{O}_{3}(200 \mathrm{~nm}$ peak to valley distance) (Dhananjay et al. 2008) are also shown in Figure 6 . These intentionally wrong inputs yield dielectric functions of DiMethoxyethyl-PTCDI with even less deviation from the actual DiMethoxyethyl-PTCDI material/ void function compared to the DiPhenyl-PTCDI material/ void function.

Based on these findings we propose a general RL model to be used instead of the 50:50 model for grown thin films:

The deposition of a large number of particles on a flat surface with random distribution of particles inside the deposition beam will yield a normal distribution, if converted to a histogram and integrated as described above, as long as surface diffusion is neglected:

$$
N(Z=k)=\frac{1}{\sqrt{2 \pi \lambda}} \exp \left(-\frac{(k-\lambda)^{2}}{2 \lambda}\right) .
$$

The value $\lambda$ is the expected value and $k$ is the number of particles sticking to the surface with the probability $N$ $(Z=k)$ at a specific area element having the size of each particle. The expected value equals the variance $E(Z)=$ $\operatorname{Var}(Z)=\lambda$. The mean square error (MSE) of this experiment is defined as

$$
\operatorname{MSE}(Z)=\frac{1}{m} \sum_{i=1}^{m}\left[k_{i}-\lambda\right]^{2},
$$

where $m$ is the number of particles which can find space on the surface, if only one particle is allowed at each area segment. The corresponding RMS function can be defined as

$$
\operatorname{RMS}(Z)=\sqrt{M S E(Z)}
$$

Each particle consumes a fraction of area of the surface. Once this area is occupied, the next monolayer starts to grow on top at this specific position. Therefore, $k$ is also equal to the number of monolayers, and, hence, $N(Z=k)$ is the probability to find $k$ monolayers at a specific position on the substrate. Then, $\lambda$ is the expected number of monolayers. For a discrete distribution as in equation (2) the variance can be calculated as follows:

$$
\operatorname{Var}(Z) \equiv \sum_{i=1}^{m}\left[Z_{i}-E(Z)\right]^{2} p_{i}
$$

Here, $p_{\mathrm{i}}=m_{\mathrm{i}} / m$ is the probability to find a particle at the position $i$ on the surface $\left(m_{\mathrm{i}}\right.$ being the number of particles at each position $i$ and $m$ the total number of particles). For a monolayer one particle is considered on each position $i$ and, hence, this ratio becomes $p_{\mathrm{i}}=1 / \mathrm{m}$. Then, equation (5) equals equation (3). Therefore, we can write $S(Z)=\sqrt{\operatorname{Var}(Z)}$. Since the particle size is small compared to the expected value a continuous distribution can be used instead of equation (2), a Gaussian distribution with $\lambda=C=\sigma^{2}$ :

$$
G(z)=\frac{1}{\sigma \sqrt{2 \pi}} \exp \left(-\frac{(z-C)^{2}}{2 \sigma^{2}}\right),
$$

where $z$ is the dimensionless height of the film. The resulting material/void function is

$$
\begin{aligned}
v(z) & =\int d z^{\prime} G(z)=\frac{1}{\sigma \sqrt{2 \pi}} \int d z^{\prime} \exp \left(-\frac{\left(z^{\prime}-C\right)^{2}}{2 \sigma^{2}}\right) \\
& =c+\frac{1}{2} \operatorname{erf}\left(\frac{z-C}{\sigma \sqrt{2}}\right) .
\end{aligned}
$$

Additionally, in the boundary condition for $z \rightarrow \infty$ only voids are remaining. This leads to $c=1 / 2$. If $C$ is defined as $50 \%$, the material/void function equals $50 \%$ at a relative depth of $z=50 \%$. This is in good agreement with $v(z)$ found from the evaluation of the AFM measurements. This also ensures that the mean amount of material in the roughness film found by the ellipsometry model equals $50 \%$ and, hence, the thickness of the $v(z)$-RL remains comparable with thicknesses determined by the common 50:50 model. Additionally, the nominal thickness of the RL can easily be defined as $d_{\mathrm{r}, \text { nom }}=d_{\mathrm{r}} / 2$, which is important for growth rate control using a quartz microbalance.

The factor $\sigma$ determines the slope of $v(z)$. A value of $\sigma=$ $13.5 \%$ matches all the empirically found material/void functions best. The resulting material/void function

$$
v(z)=50 \%\left[1+\operatorname{erf}\left(\frac{z-50 \%}{13.5 \% \sqrt{2}}\right)\right]
$$

can be included into the ellipsometry software yielding with very little deviation the same dielectric function as the Boltzmann equation discussed above derived from the various materials.

If the ellipsometry software does not allow the use of the error function a similar $v(z)$ function can also be defined based on the Boltzmann equation (1) with $A=0 \%$ 


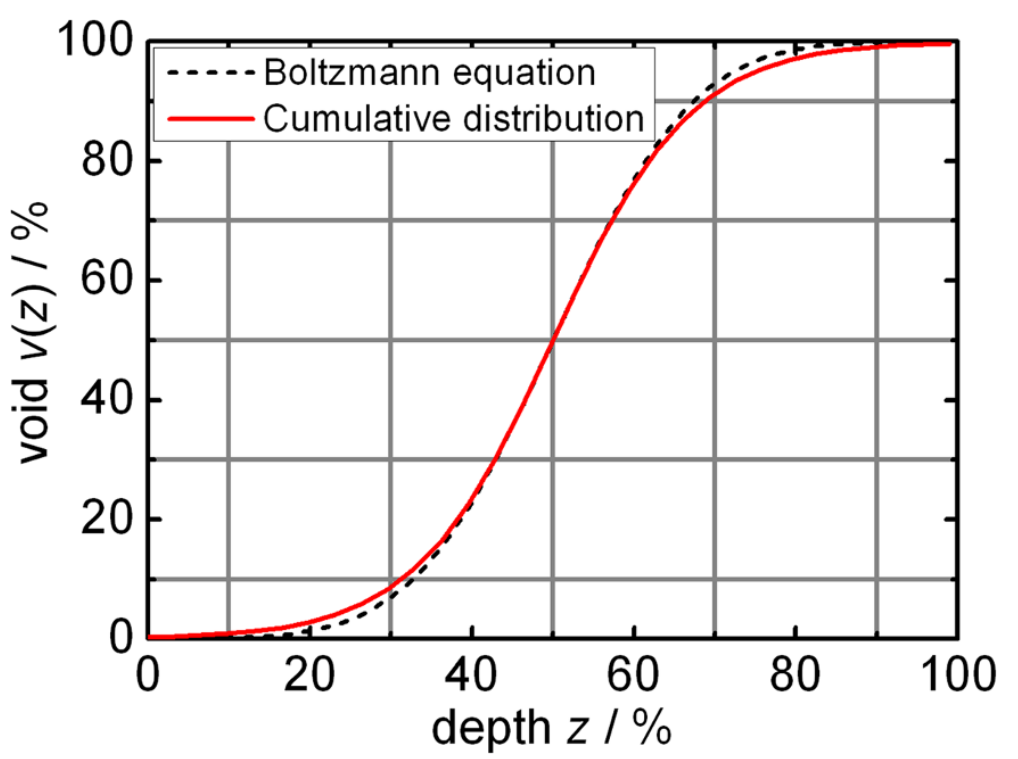

Figure 7 Comparison between proposed roughness model material/void functions $\mathbf{v}(\mathbf{z})$. The functions formularized with the Boltzmann equation and with the cumulative distribution approach yield comparable results with negligible difference with respect to the evaluation of dielectric functions.

void at the bottom of the roughness film, $B=100 \%$ void at the top of the roughness film, $C=50 \%$ material at the relative depth of $z=50 \%$, and $D=8.5 \%$ as empirically found distribution parameter. The negligible difference to $v(z)$ based on the cumulative distribution is shown in Figure 7.

Even though the equations introduce multiple new parameters, there is no additional fit parameter during SE evaluation. Only the total thickness of the RL will be fitted as with the 50:50 model before. The values $\sigma=$ $13.5 \%$ or $D=8.5 \%$, respectively, have also been used very successfully for other material systems, i.e. $\mathrm{ZnO}$ and $\mathrm{Si}$ (will be published elsewhere). There, AFM pictures were unavailable and these values had to be used as starting values. Even without freeing these parameters for fitting, the results were very satisfying. Without having a final proof, these values appear to be reliable starting values in general. Applying the model with these values, the thickness of the DiMethoxyethyl-PTCDI RL is determined to be $(94.8 \pm 0.3) \mathrm{nm}$, also without homogeneous film and with the same dielectric function.

RMS values determined by AFM measurements can be transferred into a RL thickness with $v(z)$ of equation (8) using

$$
\begin{aligned}
& R M S=\sigma \cdot d_{r}=\sqrt{\operatorname{Var}(z)}, \\
& d_{r}=\frac{R M S}{13.5 \%}
\end{aligned}
$$

It has to be pointed out that equation (10) only holds for an ideal AFM tip with a diameter of less than the smallest structure of the rough film. In the example of
DiMethoxyethyl-PTCDI the thickness of $68.4 \mathrm{~nm}$ derived from the RMS value of $9.24 \mathrm{~nm}$ deviates from the SE determined film thickness of $94.8 \mathrm{~nm}$ by a factor of 1.39. The factor can be seen as a correction factor and might deviate between different AFM tips.

The RL model will not only hold for organic thin films as described exemplarily for DiMethoxyethyl-PTCDI, but as well for all organic and inorganic films grown from a particle beam, where surface diffusion and plasmonic effects can be neglected and the counting statistical approach presented is applicable.

The RL model presented might as well be used for interfaces between two materials, if the underlying material already exhibited surface roughness before. Consequently, void has to be substituted by the material grown on top. The formalism remains the same.

Franta and Ohlídal (2005) discussed the advantages of Rayleigh-Rice theory (RRT) compared to the EMA used here, especially for laterally large irregularities (i.e. islands). Following their discussion, we also recommend to use RRT over EMA for our RL model, if this option is available. However, to our knowledge today's commercially available software does not offer a sophisticated way for implementing RRT.

\section{Conclusions}

In summary a sophisticated roughness layer model for spectroscopic ellipsometry is developed starting from empirical evaluation of AFM measurements. The model is generalized utilizing statistical considerations to be useful for a broad range of materials exhibiting high 
surface roughness. The model allows a more precise determination of the dielectric function as well as a more reliable determination of film thicknesses of highly rough materials.

\section{Endnote}

${ }^{\mathrm{a}}$ unpublished AFM investigation on $\mathrm{SiO}_{2}$ thin films by O. Zywitzki, Fraunhofer FEP Dresden.

\section{Abbreviations}

AFM: Atomic force microscopy; DiMethoxyethyl-PTCDI: N,N'-dimethoxyethyl3,4,9,10-perylene-tetracarboxylic-diimide; DiPhenyl-PTCDI: N,N'-diphenyl3,4,9,10-perylene-tetracarboxylic-diimide; EMA: Effective medium approximation; MSE: Mean square error; QMB: Quartz micro balance; RL: Roughness layer; RMS: Root mean square; RRT: Rayleigh-Rice theory; SE: Spectroscopic ellipsometry.

\section{Competing interests}

The authors declare that they have no competing interests.

\section{Authors' contributions}

DL prepared the DiMethoxyethyl-PTCDI and DiPhenyl-PTCDI thin films, measured and evaluated the ellipsometry data, evaluated the AFM measurements and developed, based on them, the ellipsometry roughness layer model. FS implemented the theoretical description starting from normal distribution, advancing the model to the use of the error function and contributing a direct translation factor for AFM measured RMS values into roughness layer thickness. DRTZ provided analysis instruments and revised the manuscript. All authors read and approved the final manuscript.

\section{Acknowledgements}

The authors are grateful to Franziska Lüttich for the AFM measurements of DiMethoxyethyl-PTCDI and DiPhenyl-PTCDI. This work was financially supported by the Scientific Priority Program 1121: Organic field-effect transistors: Structural and dynamical properties and the Bundesministerium für Bildung und Forschung (project 03IS2011 "nanett"). The publication costs of this article were funded by the German Research Foundation/DFG (Geschäftszeichen INST 270/219-1) and the Chemnitz University of Technology in the funding programme Open Access Publishing.

Received: 17 May 2013 Accepted: 4 February 2014

Published: 12 February 2014

\section{References}

Aspnes DE, Theeten JB, Hottier F (1979) Investigation of effective-medium models of microscopic surface roughness by spectroscopic ellipsometry. Phys Rev B 20:3292, doi: 10.1103/PhysRevB.20.3292

Bruggeman DAG (1935) Berechnung verschiedener physikalischer Konstanten von heterogenen Substanzen. I. Dielektrizitätskonstanten und Leitfähigkeiten der Mischkörper aus isotropen Substanzen. Ann Phys 416:636-679, doi:10.1002/andp.19354160705

Dhananjay, Cheng S-S, Yang C-Y, Ou C-W, Chuang Y-C, Wu MC, Chu C-W (2008) Dependence of channel thickness on the performance of $\ln _{2} \mathrm{O}_{3}$ thin film transistors. J Phys D Appl Phys 41:092006, doi:10.1088/0022-3727/41/9/092006

Franta D, Ohlídal I (2005) Comparison of effective medium approximation and Rayleigh-Rice theory concerning ellipsometric characterization of rough surfaces. Opt Commun 248:459, doi:10.1016/j.optcom.2004.12.016

Gordan OD, Sakurai T, Friedrich M, Akimoto K, Zahn DRT (2006a) Ellipsometric study of an organic template effect: $\mathrm{H}_{2}$ PC/PTCDA. Org Electron 7:521-527, doi:10.1016/j.orgel.2006.07.008

Gordan OD, Hermann S, Friedrich M, Zahn DRT (2006b) Optical properties of the interfaces in organic/organic multilayered heterostructures. J Phys IV France 132:73-76, doi:10.1051/jp4:2006132014

Lehmann D, Zahn DRT (2009) The electrical and dielectrical behavior of n-conducting perylene tetracarboxylic diimide derivatives. Appl Phys A 95:203-207, doi:10.1007/s00339-008-5020-2

Louis JS, Lehmann D, Friedrich M, Zahn DRT (2007) Study of dependence of molecular orientation and optical properties of zinc phthalocyanine grown under two different pressure conditions. J Appl Phys 101:013503, doi:10.1063/1.2403845

Méndez-Vilas A, Nuevo MJ, González-Martín ML, Labajos-Broncano L (2002) Quantitative surface roughness determination of materials by AFM: some limitations. Mater Sci Forum 408-412:245, doi:10.4028/www.scientific.net/ MSF.408-412.239

Volintiru I, Creatore M, van de Sanden MCM (2008) In situ spectroscopic ellipsometry growth studies on the Al-doped $\mathrm{ZnO}$ films deposited by remote plasma-enhanced metalorganic chemical vapor deposition. J Appl Phys 103:033704, doi:10.1063/1.2837109

doi:10.1186/2193-1801-3-82

Cite this article as: Lehmann et al.: Thin films with high surface roughness: thickness and dielectric function analysis using spectroscopic ellipsometry. SpringerPlus 2014 3:82.

\section{Submit your manuscript to a SpringerOpen ${ }^{\odot}$ journal and benefit from:}

- Convenient online submission

- Rigorous peer review

- Immediate publication on acceptance

- Open access: articles freely available online

- High visibility within the field

- Retaining the copyright to your article

Submit your next manuscript at springeropen.com 